\title{
Un nouveau système d'extraction d'œufs de diptères du sol. Le séparateur à turbulence
}

\author{
Kouame N'GUETTA \& Etienne BRUNEL* \\ I.N.R.A., Station de Zoologie et de Lutte biologique, B.P. 2078, F 06606 Antibes \\ * Laboratoire de Zoologie, B.P. 29, Centre de Recherches de Rennes, F 35650 Le Rheu
}

\begin{abstract}
Les auteurs décrivent un nouveau dispositif pour extraire du sol les œufs de diptères lorsque la matière organique est importante. Les résultats montrent que, avec le séparateur à turbulence, le rendement de l'opération (pourcentage de récupération des œufs) est augmenté de $16 \mathrm{p} .100$ pour un gain de temps de $75 \mathrm{p}$. 100 . Il est d'autre part plus regulier que celui de la methode de l'elutriateur de Kort utilise seul.
\end{abstract}

Mots clés additionnels : Extraction, ceufs, diptères, Opomyzidae.

The authors describe a new apparatus for extracting the eggs of flies from soil when the samples are full of organic matter. With this turbulent separator, egg extraction could be increased by $16 \%$, with a $75 \%$ gain in time and more regular results than with the classic kort elutriator.

Additional key words : Extraction, eggs, Diptera, Opomyzidae.

\section{INTRODUCTION}

Geomyza tripunctata Fall (Diptère, Opomyzidae) s'attaque à de nombreuses graminées et aux cultures de maïs et de ray-grass (Lolium perenne et $L$. italicum) dans l'ouest de la France (BRUNEL, 1974). Des travaux de biologie nécessitent l'obtention d'œufs viables, c'est-à-dire ayant subi le moins de dommages possible et susceptibles de donner des larves capables de se développer. Or la mouche dépose ses œufs au collet de la plante soit au niveau du sol soit légèrement en dessous. Ceux-ci de taille réduite $(0,8$ à $1 \mathrm{~mm}$ de long, $0,2 \mathrm{~mm}$ de diamètre) sont difficilement visibles à l'œil nu.

La récolte des œufs peut se faire par des méthodes mécaniques qui font appel à la différence de densité entre l'œuf et l'eau. La méthode de flottation a été utilisée pour la première fois par NORMAND (1911), puis a subi des modifications (MORRIS, 1922 ; LADELL, 1936 ; D'AGUILAR et al., 1957 ; HALE, 1964 ; AUCAMP
\& RYKE, 1964 ; HEALEY \& RUSSEL-SMITH, 1970) pour s'adapter au matériel biologique. La méthode de HEALEY, qui emploie un mélange d'eau et de glycérine (densité 1,4) a été reprise par BAILLIOT \& DELETTRE (1972) pour extraire du sol des larves de diptères.

D'autre part, on utilise couramment au laboratoire l'élutriateur de KORT (KORT, 1960), pour l'extraction de larves et de kystes de nématodes. Ce procédé semble convenir également à l'extraction d'œufs de $G$. tripunctata. Il est plus rapide que celui utilisé par BARDNER et al. (1973) pour l'extraction des œufs d'Hylemyia coarctata et celui de BAILliot \& DELETTRE (1972) et TREHEN et al. (1975).

Le principe est basé sur la différence de densité entre les différents éléments et l'eau. Les éléments lourds se déposent au fond d'un cylindre, les œufs et les matières organiques légères sont recueillies par flottation sur un tamis à mailles fines $(0,25 \mathrm{~mm})$. L'obtention des œufs nécessite l'examen de la matière organique millimètre par millimètre et demande pour un seul échantil- 
lon environ $2 \mathrm{~h}$ de travail alors que plusieurs échantillons doivent être examinés dans la journée. De plus, cette dernière opération ne permet pas de trouver tous les œufs ; près de 40 p. 100 étant cachés et jetés avec les résidus organiques. La méthode de centrifugation permettrait d'obtenir aisément le maximum d'œufs mais présente l'inconvénient de les détériorer.

\section{DISPOSITIF PROPOSÉ}

Nous proposons un dispositif qui présente l'avantage d'éliminer une grande partie de la matière organique obtenue par l'élutriateur de KORT et de réduire le temps de récupération des œufs.

\section{A. Principe du séparateur à turbulence}

Le principe de fonctionnement est identique à celui de l'élutriateur de KORT. Un mélange de matières organiques et d'œufs est mis en solution dans un entonnoir et brassé dans une turbulence provoquée par la rencontre de 2 courants ascendant et descendant d'eau. Ceci permet la séparation des différents éléments, d'où le nom du dispositif : séparateur à turbulence. L'interruption de la turbulence entraîne la décantation des éléments de densité supérieure à celle de l'eau. Les œufs sont toujours recueillis par flottation.

\section{B. Composition de l'appareil (fig. 1)}

Le dispositif du séparateur à turbulence est composé :

- d'un entonnoir qui reçoit le matériel recueilli à l'élutriateur de KORT ;

- d'un réservoir relié à l'entonnoir par un tuyau souple ;

- ce réservoir se prolonge dans sa partie inférieure par un autre tuyau souple et possède un orifice latéral dans sa partie supérieure ;

- 2 robinets sont respectivement placés en amont (sur le tuyau souple supérieur) et en aval du réservoir ;

- l'arrivée d'eau se divise en 2 branches dont l'une permet de remplir ou de nettoyer l'entonnoir et l'autre arrivant à l'ouverture latérale du réservoir permet de remplir ce dernier ainsi que l'entonnoir, par le bas. Le débit du dispositif est de $2,4 \mathrm{l} / \mathrm{min}$;

-2 robinets situés respectivement sur ces 2 tuyaux permettent essentiellement de régler le débit d'eau arrivant dans le réservoir ;

- un robinet gouvernant l'arrivée générale de l'eau permet de mettre l'appareil en marche.

\section{Fonctionnement du séparateur à turbulence (fig. 1)}

Les robinets R4 ouvert et R5 fermé, on met l'appareil en marche en ouvrant le robinet $\mathrm{R} 1$. L'eau admise

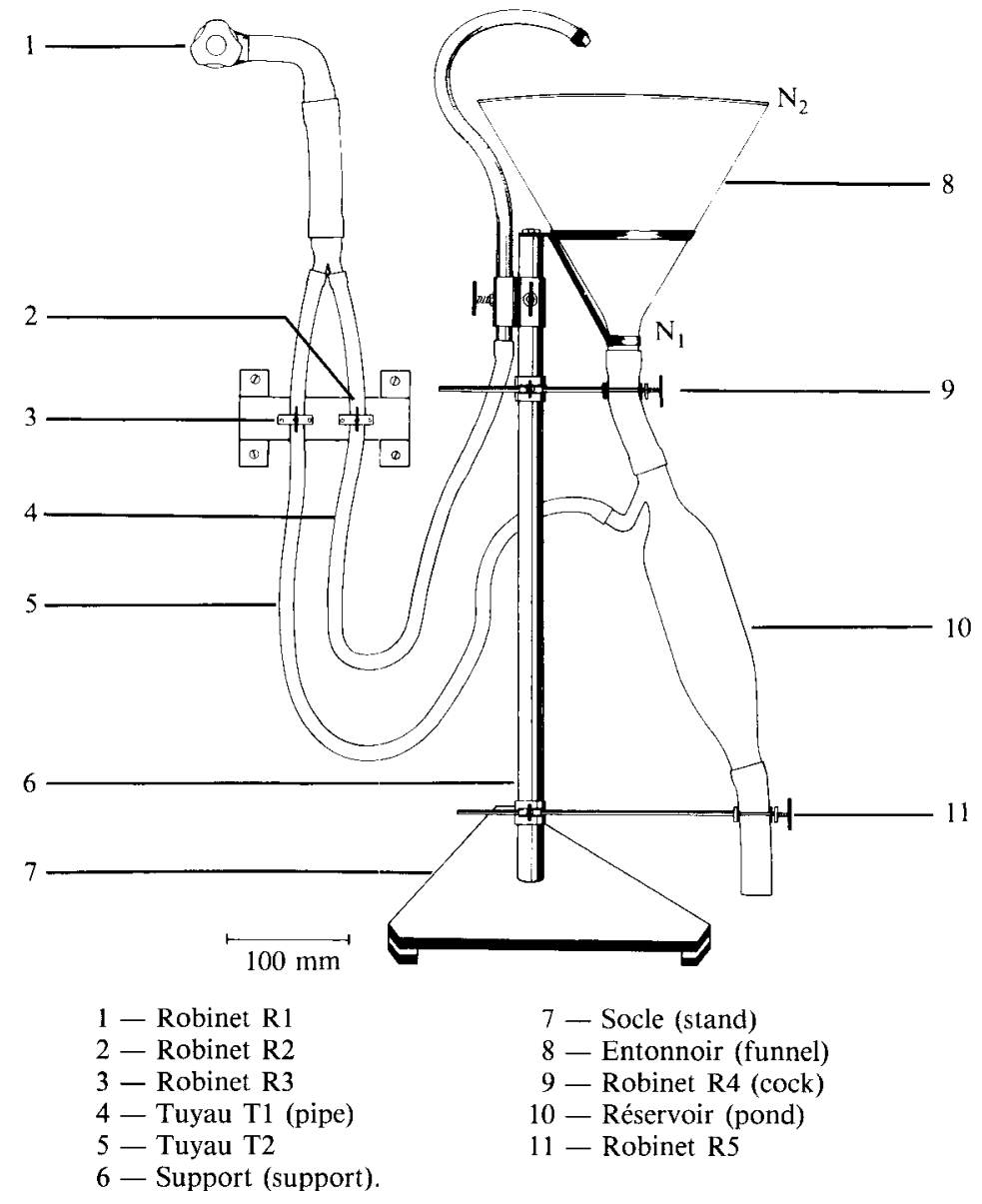

Figure 1

Le séparateur à turbulence.

The turbulent separator. 
TABLEAU 1

Comparaison entre la méthode de tri manuel et celle utilisant le séparateur à turbulence. Comparison between manual sorting and use of the turbulent separator.

\begin{tabular}{|c|c|c|c|c|c|c|}
\hline Méthodes & \multicolumn{2}{|c|}{ Nombre d'œufs } & $\begin{array}{c}\text { Rendement } \\
\% / 0\end{array}$ & $\begin{array}{l}\text { Rendement } \\
\text { moyen } \% 0\end{array}$ & $\begin{array}{l}\text { Durée } \\
\text { (min) }\end{array}$ & $\begin{array}{l}\text { Durée moyenne } \\
\text { (min) }\end{array}$ \\
\hline Tri manuel & $\begin{array}{r}47 \\
50 \\
90 \\
100\end{array}$ & $\begin{array}{l}31 \\
28 \\
70 \\
67\end{array}$ & $\begin{array}{l}66 \\
56 \\
78 \\
67\end{array}$ & 67 & $\begin{array}{r}87 \\
91 \\
140 \\
127\end{array}$ & 111 \\
\hline Tri au moyen du séparateur & $\begin{array}{r}47 \\
50 \\
90 \\
100\end{array}$ & $\begin{array}{l}39 \\
41 \\
77 \\
83\end{array}$ & $\begin{array}{l}83 \\
82 \\
85 \\
83\end{array}$ & 83 & $\begin{array}{l}33 \\
42 \\
45 \\
49\end{array}$ & 42 \\
\hline
\end{tabular}

se divise dans les tuyaux $\mathrm{T} 1$ et $\mathrm{T} 2$. Les robinets $\mathrm{R} 2$ et R3 étant ouverts, on remplit le réservoir et l'entonnoir à l'aide de $\mathrm{T} 1$ et T2. Lorsque l'eau dans l'entonnoir atteint le niveau $\mathrm{N} 1$, on y ajoute le mélange à séparer.

Le courant d'eau ascendant provoqué par l'eau arrivant de $\mathrm{T} 2$ et celui descendant de $\mathrm{T} 1$ créent une turbulence, ce qui brasse le mélange et sépare les différentes particules. La variation des débits d'eau obtenue à l'aide des robinets R2 et R3 permet de jouer sur la vitesse de décantation des particules. Lorsque le niveau d'eau dans l'entonnoir atteint $\mathrm{N} 2$, soit à $1 \mathrm{~cm}$ du bord supérieur, on supprime le remplissage du côté supérieur de l'entonnoir et on réduit l'arrivée de l'eau de T2. Ceci a pour effet de ralentir l'arrivée de l'eau de T2 dans l'entonnoir, de provoquer la descente dans le réservoir des particules lourdes. La vitesse de décantation est maximum lorsque l'on ferme complètement R3. Plus les éléments à récupérer sont lourds, plus il est recommandé d'utiliser une vitesse de décantation faible.

Lorsque la séparation est terminée (au bout de $5 \mathrm{~min}$ à $10 \mathrm{mn}$ selon le cas), on ferme R4 puis on ouvre R5 et R3. Le courant d'eau arrivant de T2 permet de chasser le dépôt de matière organique du réservoir. On ouvre ensuite R4 pour récupérer le contenu de l'entonnoir sur le tamis à mailles fines $(0,25 \mathrm{~mm})$. On ouvre $\mathrm{R} 2$ pour nettoyer l'entonnoir au moyen du tuyau T1. L'appareil est arrêté lorsqu'on ferme R1. Les œufs recueillis sont ensuite comptés sous une loupe binoculaire.

\section{Evaluation de l'intérêt pratique du séparateur à turbulence}

Il s'agit de comparer les rendements (pourcentage d'œufs récupérés) ainsi que les temps de travail nécessaires des 2 méthodes : élutriateur de KORT et élutriateur de KORT + séparateur à turbulence.

Deux lots d'œufs, provenant d'un tri précédent, dont le nombre est inconnu de l'opérateur, sont respectivement mélangés à 2 lots de même quantité de matière organique (exempts d'œufs) extraits du KORT issue d'un pot de blé non présenté à la ponte de Geomyza. L'un est directement trié sous une loupe binoculaire et l'autre passé au séparateur à turbulence.
Le temps mis pour chaque opération ainsi que les nombres d'œufs obtenus sont notés à la fin de chaque manipulation (tabl. 1).

Les conditions de récupération des œufs sont les mêmes sans que puisse intervenir le problème de l'adhérence des œufs sur le substrat végétal. Un contrôle des plantes après passage à l'élutriateur nous a montré que ce cas ne se présentait pas pour les œufs de Geomyza.

La comparaison des résultats est à l'avantage du séparateur à turbulence. Le rendement, taux de récupération des œufs, passe de 67 p. 100 à 83 p. 100. La durée des opérations de tri manuel est réduite de $111 \mathrm{~min}$ à $40 \mathrm{~min}$ soit un gain de temps de 75 p. 100 .

Les œufs obtenus sont viables. Ils ont permis la mise en place d'essais insecticides et l'étude des durées de développement des différents stades de G. tripunctata (N'GUETTA, 1986).

\section{CONCLUSION}

La plupart des méthodes utilisées pour l'extraction des différents stades d'insectes du sol sont basées sur le principe de flottation, après élimination des éléments grossiers.

Dans des échantillons où la matière organique est peu abondante, la technique de l'élutriateur de KORT est facile d'emploi. Cependant dans de nombreux sols, en particulier dans les zones de culture la matière organique est souvent abondante et devient par conséquent un obstacle à l'extraction et à une estimation des populations d'œufs. Le séparateur à turbulence présente l'avantage, tout en réduisant le temps de manipulation, d'apporter une plus grande précision.

Les résultats obtenus montrent que ce dispositif pourrait également être utilisé pour extraire des œufs, des larves et des pupes d'autres diptères à développement édaphique comme Delia radicum B., Psila rosae Fab., Delia platura Meig., H. coarctata Fall., etc. ainsi que des kystes ou des larves de nématodes.

Reçu le 8 octobre 1987. Accepté le 14 février 1988. 


\section{RÉFÉRENCES BIBLIOGRAPHIQUES}

Aucamp J. L., Ryke P. A. J., 1964. Preliminary report on a grease film extraction. Method for soil micro-arthropods. Pedobiologia, 4, 77.79.

Bailliot S., Delettre Y., 1972. Contribution à l'étude écologique des diptères à larves édaphiques. DEA écologie animale, Université de Rennes, $77 \mathrm{p}$.

Bardner R., Fletcher K. E., Jones M. G., Lofty J. R., 1973. Fluctuations in populations of wheat bulb fly (Leptohylemyia coarctata) at Rothamsted. Ann. appl. Biol., 74 (1), 25-34.

Brunel E., 1974. Un nouveau ravageur observé dans l'ouest de la France : Geomyza tripunctata Fall. (Diptère, Opomyzidae). C. $R$. Acad. Agric., 3, 197-202.

D'aguillar J., Benard R., Bessard A., 1957. Une méthode de lavage pour l'extraction des arthropodes terricoles. Ann. Ephiphyt., 1, 91 99.

Hale W. G., 1964. A flotation method for extracting Collembola from organic soils. J. Anim. Ecol., 33, 363-369.
Healey I. N., Russel-Smith A., 1970. Extraction of fly larvae from woodland soils. Soil. Biol. Biochem., 2, 119-129.

Kort J., 1960. A technique for the extraction of Heterodera cysts from wet soil and for the estimation of their egg and larval content. Versl. Meded. plziektenk. Dienst. Wageningen, 233, 6 p.

Ladell W. R. S., 1936. A new apparatus for separating insects and other arthropods from the soil. Ann. appl. Biol., 23, 862-879.

Morris H. M., 1922. On a method of separating insects and other arthropods from soil. Bull. ent. Res., 13, 197-200.

N'guetta K., 1986. Contribution à l'étude de la biologie de la Mouche $d u$ poireautage du maïs: Geomyza tripunctata Fall. DAA Protection des cultures, ENSA Rennes, $37 \mathrm{p}$.

Normand P., 1911. Ein neue Sammeltechnik fur Subterrankäfer (Schwenn-Methode). Coleoptologische Rundschan, 8-9, 119-124.

Trehen P., Bailliot S., Delettre Y., 1975. Introduction à la dynamique des populations de Diptères dans les sols de la lande rase armoricaine de la région de Paimpont. Rev. Ecol. Biol. Sol., 12, 101-112. 